\title{
Protective effect of polysaccharide peptide on cerebral ischemia-reperfusion injury in rats
}

\author{
PENGCHENG XING ${ }^{1}, \mathrm{KE} \mathrm{MA}^{1}, \mathrm{JUN}^{\mathrm{W}} \mathrm{WU}^{2}, \mathrm{WEI} \mathrm{LONG}^{3}$ and DONGLIAN WANG ${ }^{1}$ \\ ${ }^{1}$ Department of Emergency; ${ }^{2}$ ICU; ${ }^{3}$ Department of Geriatric Medicine, Shanghai Sixth People's Hospital East Area \\ Affiliated to Shanghai University of Medicine and Health Sciences, Shanghai 201306, P.R. China
}

Received December 8, 2017; Accepted September 6, 2018

DOI: $10.3892 / \mathrm{mmr} .2018 .9579$

\begin{abstract}
In the present study, the protective effects and regulatory mechanism of polysaccharide peptide (PSP) were investigated in rats with cerebral ischemia-reperfusion (IR) injury. Neuroblastoma N2a cells were divided into five groups: Negative control; IR injury; PSP low dose treatment; PSP middle dose treatment; and PSP high dose treatment. In vitro, the cell viability was detected by an MTT assay. ELISA was performed to determine the activity of lactate dehydrogenase (LDH) and caspase-3. A cerebral IR injury model in vivo was established, and hematoxylin and eosin (H\&E) staining, western blotting, neurological deficit score and cerebral infarction were assessed. The cell viability was markedly improved following treatment with PSP and the activity of LDH and caspase-3 was decreased following PSP administration $(\mathrm{P}<0.05)$. The in vivo studies determined that the neurological deficit score and cerebral infarction volume were reduced with the concentration of PSP increasing between 150 and $250 \mathrm{mg} / \mathrm{kg}$. The H\&E staining indicated that PSP was able to protect the nerve cells against the cerebral IR injury. In addition, PSP upregulated the decreased silent information regulator protein 1 , peroxisome proliferator-activated receptor $\gamma$ coactivator- $1 \alpha$ and apoptosis regulator B-cell lymphoma 2 expression induced by cerebral IR injury. The
\end{abstract}

Correspondence to: $\mathrm{Dr} \mathrm{Ke} \mathrm{Ma}$, Department of Emergency, Shanghai Sixth People's Hospital East Area Affiliated to Shanghai University of Medicine and Health Sciences, 222 West Three Road Around Lake, Nanhui New City Town, Pudong, Shanghai 201306, P.R. China

E-mail:ma_ke2001@sina.com

Abbreviations: IR, ischemia-reperfusion; PSP, polysaccharide peptide; $\mathrm{LDH}$, lactate dehydrogenase; CVD, cerebrovascular disease; ICVD, ischemic cerebrovascular disease; ROS, reactive oxygen species; TCM, traditional Chinese medicine; NC, negative control; TTC, triphenyl tetrazolium chloride; TBS, Tris-buffered saline; SIRT1, silent information regulator protein 1; PGC-1 $\alpha$, peroxisome proliferator-activated receptor $\gamma$ coactivator- $1 \alpha$

Key words: PSP, cerebral IR, apoptosis, SIRT1/PGC-1 $\alpha$ signaling pathway, neuroprotective effect protein expression level of caspase-3 and apoptosis regulator apoptosis regulator $\mathrm{Bcl}-2$-like protein 4 was downregulated following PSP administration. These results suggested that PSP may improve nerve cell viability, enhance the neuroprotective role in cerebral IR injury and provide a novel approach for the treatment of cerebral IR injury.

\section{Introduction}

Cerebral ischemia describes the situation in which blood flow to the brain is insufficient in meeting metabolic demand. This results in cerebral hypoxia, the death of brain tissue, cerebral infarction or ischemic stroke $(1,2)$. Cerebral ischemia is associated with cerebrovascular disease or disorder (CVD), which is a common disease with high morbidity and mortality. Ischemic cerebrovascular disease (ICVD) has a high incidence and high recurrence rate accounting for $70 \%$ of total cerebrovascular disease, which is clinically associated with myocardial infarction, thrombolytic therapy, coronary angioplasty, coronary revascularization and heart transplantation (3) Although it is widely accepted that blood reflow following ischemia is important for increasing cardiomyocyte survival to minimize the cardiac dysfunction and myocardial damage, reperfusion itself additionally aggravates myocardial injury (4). Therefore, the prevention and treatment of cerebral ischemia-reperfusion (IR) injury is becoming increasing important in ICVD therapy.

However, the exact pathogenesis of cerebral IR injury remains unknown. Cumulative evidence suggests that numerous factors are involved in ischemic brain injury, including apoptosis, acid poisoning, ionic imbalance, excitotoxicity, oxidative stress, peri-infarct depolarization, nutritive stress, inflammation and reactive oxygen species (ROS) (5-12). Furthermore, numerous signaling pathways have been implicated in the pathogenesis of cerebral IR injury, including mitogen-activated protein kinases $(13,14)$. Additionally, during ischemic brain damage, the cell metabolism abnormalities may be derived from the interrupted blood, oxygen and glucose supply, which ultimately result in neuronal death or apoptosis (2). Therefore, anti-inflammation and anti-oxidant treatment strategies are being developed to treat cerebral IR injury.

Due to the complex alterations associated with IR injury, it has become difficult to identify a novel pharmacological 
drug with a protective effect. Previous studies preferentially developed therapeutics for IR injury based on the advantages of traditional Chinese medicine (TCM) $(15,16)$. Seaweed is one of the most abundant sources of polysaccharides used in TCM, with alginate, agar, fucoidan, agarose and carrageenan all present (16). A previous study demonstrated that M. charantia polysaccharide (MCP) could be a promising neuroprotective ingredient of $\mathrm{M}$. charantia and its mechanisms could be at least in part attributed to its antioxidant activities and its inhibition of JNK3 signaling cascades during cerebral ischemia/reperfusion injury in a rat model (17). Zhou et al (18) reported that treatment with Ganoderma lucidum polysaccharides, aloe polysaccharide, ginkgo leaf tablet and nimodipine following cerebral ischemic injury significantly reduced caspase-3 protein and mRNA expression levels in the cerebral cortex compared with the cerebral ischemia/reperfusion Sprague-Dawley (SD) rat model group, and were significantly increased compared with the sham surgery group $(\mathrm{P}<0.05)$. Lu et al (19) revealed that the protective effect of aloe polysaccharide on cerebral ischemia may be due to the inhibition of neuronal cell apoptosis via downregulation of caspase-3 protein expression. Agaricus brasiliensis polysaccharides may affect malondialdehyde and superoxide dismutase activity, and caspase-3 level in IR rats, resulting in cardiovascular protection (20). The effect of polysaccharide peptide (PSP) on cerebral injury caused by IR remains unknown, even though substantial research and numerous studies of the neuroprotective effect of the polysaccharides have been performed. Additionally, the mechanism requires further clarification.

At present, the study of cerebral IR injury has attracted considerable research interest. Cerebral ischemia leads to serious damage of local tissue and brain function. The degree of damage is associated with the duration of ischemia and the remaining blood flow. Severe ischemia with a longer duration may lead to infarction. The primary therapeutic method is IR, allowing ischemia brain tissue access to a supply of oxygen and the necessary nutrients again. However, the therapeutic efficacy of reperfusion is undesirable. Cerebral IR may result in neuron death due to an increase in the generation of free radicals caused by IR injury (21). Furthermore, reperfusion may lead to a reduction in the content of excitatory amino acid and alteration of the ultramicrostructure of brain tissue, including mitochondrial swelling, calcium deposition and endothelial swelling (1). Therefore, in the present study, the neuroprotective action and underlying mechanisms of PSP against cerebral IR-induced injury were investigated in vitro and in vivo.

\section{Materials and methods}

Cell culture. Mouse neuroblastoma cell line N2a was purchased from BeNa Culture Collection (Jiangsu, China) and incubated in culture medium (Dulbecco's modified Eagle's medium and opti-Minimum Essential Medium 1:1, both from Gibco; Thermo Fisher Scientific, Inc., Waltham, MA, USA) containing $10 \%$ fetal bovine serum (Hyclone; GE Healthcare Life Sciences, Logan, UT, USA) in a $5 \% \mathrm{CO}_{2}$ incubator at $37^{\circ} \mathrm{C}$. The medium was replaced with fresh medium every two days. The cells were used for propagation or experimental use when $70-80 \%$ confluent.
Establishment of the model of IR in vitro. The cells were divided into five groups; Negative control (NC) group, IR injury group, PSP low dose treatment group (PSP1; $110 \mathrm{mg} / \mathrm{ml}$ ), PSP middle dose treatment group (PSP2; $30 \mathrm{mg} / \mathrm{ml}$ ) and PSP high dose treatment group (PSP3; $50 \mathrm{mg} / \mathrm{ml}$ ).

When the cells were cultured to $80 \%$ confluence, the cells in the NC group were continually cultured in complete culture solution, while the cells in the IR and PSP groups were treated with an equal volume of equilibrated medium, containing $116 \mathrm{mM} \mathrm{NaCl}, 5.4 \mathrm{mM} \mathrm{KCl}, 0.8 \mathrm{mM} \mathrm{MgSO}_{4}, 1 \mathrm{mM} \mathrm{NaH}_{2} \mathrm{PO}_{4}$, $0.9 \mathrm{mM} \mathrm{CaCl}_{2}$ and $10 \mathrm{mg} / \mathrm{l}$ phenol red. The cells were incubated in $5 \% \mathrm{CO}_{2}$ and $95 \% \mathrm{~N}_{2}$ for $30 \mathrm{~min}$ at $37^{\circ} \mathrm{C}$, and subsequently the equilibrated medium was replaced with complete culture solution. Different concentrations of PSP (Xi'an Tianrui Biological Technology Co., Ltd. Xi'an, China) were added to the PSP groups.

MTT assay. The cells were seeded on a 96-well plate at a density of $10^{5}$ cells/well and incubated in $5 \% \mathrm{CO}_{2}$ at $37^{\circ} \mathrm{C}$. Following cell growth to $80 \%$, different concentrations of PSP were added to the wells. After $24 \mathrm{~h}, 10 \mu \mathrm{l}(1 \mathrm{~g} / \mathrm{l})$ of MTT solution (Gibco; Thermo Fisher Scientific, Inc.) was added into each well and cultured for $4 \mathrm{~h}$ at $37^{\circ} \mathrm{C}$. The supernatant was removed and $150 \mu \mathrm{l}$ dimethyl sulfoxide was added. The optical (OD) was measured with the multifunctional microplate reader SpectraMax M5 (Molecular Devices, LLC, Sunnyvale, CA, USA) at $490 \mathrm{~nm}$. Each group was conducted in six parallel experiments.

Lactate dehydrogenase ( $\mathrm{LDH}$ ) activity determination. The medium was collected and centrifuged at $4^{\circ} \mathrm{C}$ and $380 \mathrm{x} \mathrm{g}$ for 5 min to obtain the supernatant. The LDH activity was determined by ELISA. The experimental process was completely performed according to the protocols of an LDH kit (AB102526; Abcam, Cambridge, UK). The OD value of LDH protein was measured using a microplate reader at $450 \mathrm{~nm}$. The standard curve was established, according to the concentration and $\mathrm{OD}$ value. The experiment was repeated three times.

Detection of caspase-3 activity in cells. The cells were harvested and lysed in RIPA buffer (Beyotime Institute of Biotechnology, Haimen, China) on ice for $20 \mathrm{~min}$. Following centrifugation at $44^{\circ} \mathrm{C}$ and $380 \mathrm{x}$ g for $5 \mathrm{~min}$, the supernatant was collected and the total proteins were quantified, according to a caspase-3 activity assay kit (Jiancheng Bioengineering Institute, Nanjing, China). The measurement of the caspase-3 activity was processed in protein analytic buffer $(20 \mathrm{nmol} / 1$ HEPES, $2 \mathrm{mmol} / 1$ dithiothreitol, $10 \%$ glycerol and $20 \mu \mathrm{mol} / 1$ DEVD-pNA) at $37^{\circ} \mathrm{C}$ for $2 \mathrm{~h}$. The OD value of the sample was measured at $405 \mathrm{~nm}$ using a microplate reader. The content of caspase- 3 was calculated using the standard curve. The experiment was repeated three times.

Animals. A total of 75 healthy male SD rats (10-12 weeks; 200-250 g) were purchased from The Experimental Animal Center of Guangzhou University of Traditional Chinese Medicine (Guangzhou, China). All rats were kept in controlled conditions at $25 \pm 3^{\circ} \mathrm{C}$ and $35-60 \%$ relative humidity. A 12-h light/dark cycle was maintained. All the animals were allowed to acclimate to laboratory conditions for $\geq 1$ week prior to the 
experiment and had free access to water and food. All the animal works were performed stringently, according to the regulation of the Guide for the Care and Use of Laboratory Animals in China (22). The protocol was approved by the Committee of the Ethics of Animal Experiments of Shanghai Jiaotong University Affiliated Sixth Hospital Provincial Centre for Disease Control and Prevention (proposal authorization no. SYXK/2016/050122). All surgeries were conducted using appropriate anesthesia and all efforts were made to reduce the pain and suffering of the animals.

In vivo IR model. The rats were randomly divided into five groups; sham operation group (sham; $n=15)$, IR injury group $(\mathrm{n}=15)$, PSP1 (150 mg/kg; n=15), PSP2 (200 mg/kg; $\mathrm{n}=15)$ and PSP3 (250 mg/kg; $\mathrm{n}=15)$. First, $10 \%$ chloral hydrate (Sinopharm Chemical Reagent Co., Ltd., Shanghai, China) was injected intraperitoneally into rats at a dose of $300-350 \mathrm{mg} / \mathrm{kg}$. There were no significant signs of peritonitis. Following anesthesia, the model of the right middle cerebral artery occlusion was selected for further experimental use. In the sham group, the right common carotid artery, external carotid artery and internal carotid artery were separated without a plug bolt. The plug was pulled out in the other groups after $2 \mathrm{~h}$ of ischemia, followed by reperfusion for $24 \mathrm{~h}$. The low, middle and high dose group rats received 150,250, $250 \mathrm{mg} / \mathrm{kg}$, respectively, via intraperitoneal injection at $2 \mathrm{~h}$ after ischemia; the sham group and IR group were intraperitoneally injected with an equal volume of saline solution at the same time point.

Neurological assessment. The neurological assessment was processed, according to the Zea Longa method (23). The neurological deficiency was assessed consistently according to a 5-point scale system: 0 , no neurological deficit; 1 , the contralateral torso and forelimb may not be thoroughly stretched; 2 , when the tail was held, the animal may be turned to the ipsilateral side; 3 , no spontaneous motor activity or falling to the left side; 4, unable to walk or loss of consciousness. Each experiment was repeated three times.

Calculation of cerebral infarction volume. The area of cerebral infarction was identified using triphenyl tetrazolium chloride (TTC; Sigma-Aldrich; Merck KGaA, Darmstadt, Germany) staining. Following the neurological assessment grading, five rats in each group were intraperitoneally injected with $10 \%$ chloral hydrate. The brains were collected following anesthesia and decapitation, and were subsequently placed in $-20^{\circ} \mathrm{C}$ for $15 \mathrm{~min}$. The tissue slices were processed to $\sim 1 \mathrm{~mm}$ thickness using a cryostat microtome. The slices were placed in $2 \%$ TTC solution, incubated at $37^{\circ} \mathrm{C}$ for $30 \mathrm{~min}$, and were subsequently fixed in $4 \%$ paraformaldehyde at $4^{\circ} \mathrm{C}$ for $24 \mathrm{~h}$. Images of the slices were captured, the infarct area in each section was calculated using an image analyzer (Image-Pro Plus version 6.0; Media Cybernetics, Inc., Rockville, MD, USA). and the cerebral infarction volume percentage was calculated using the formula:

Infarction volume $(\%)=$ right cerebral infarction volume $x \frac{\text { slice thickness }}{\text { right brain tissue volume }} \times 100$

The infarcted volume was calculated as a percentage of the total brain area.
Histopathological staining analysis. Male Sprague-Dawley rats were injected intraperitoneally with $10 \%$ chloral hydrate (300-350 mg/kg body weight; Sinopharm, Chemical Reagent Co., Ltd.), and the hearts were exposed through thoracotomy following anesthesia. The needle was inserted from the apical position, the right atrial appendage vein was cut open and $0.9 \%$ saline solution was rapidly perfused in the heart until the clear liquid outflowed from right atrial appendage, subsequently $4 \%$ paraformaldehyde (Sinopharm, Chemical Reagent Co., Ltd.) was perfused to the heart followed by quick decapitation and collection of the brain, which was fixed at $4{ }^{\circ} \mathrm{C}$ for $24 \mathrm{~h}$ with $4 \%$ paraformaldehyde. The coronal brain tissue was cut into slices of $3 \mathrm{~mm}$ thickness followed by routine embedding in paraffin. The brains were subsequently sliced to $5 \mu \mathrm{m}$ thickness, dewaxed with xylene and hydrated using a series of graded concentrations of ethanol (100\% ethanol, 5 min; $95 \%$ ethanol, 1 min; $80 \%$ ethanol, 5 min; $75 \%$ ethanol, $5 \mathrm{~min}$; distilled water, $2 \mathrm{~min}$ ). Hematoxylin and eosin (H\&E) staining was performed using the routine method at room temperature for $12 \mathrm{~min}$. Following dehydration, sections were treated with xylene at room temperature for 10 min twice. The tissue sections was examined using a light microscope (OriGene Technologies, Inc.) to examine the histopathological morphology at a magnification of $\mathrm{x} 100$.

Western blotting. Western blotting was performed to determine the protein expression of silent information regulator protein (SIRT1), peroxisome proliferator-activated receptor $\gamma$ coactivator- $1 \alpha$ (PGC-1 $\alpha$ ), caspase-3, B-cell lymphoma 2 (Bcl-2) and Bcl-2-like protein 4 (Bax), according to a standard protocol. The brain tissues were lysed on ice and centrifuged at $4^{\circ} \mathrm{C}$ and $13,680 \mathrm{x} \mathrm{g}$ for $20 \mathrm{~min}$. The supernatant was extracted using RIPA buffer and the protein concentration was measured using a bicinchoninic acid protein quantification kit. Total proteins $(20 \mu \mathrm{g})$ were loaded and separated by $12 \%$ SDS-PAGE, and transferred to polyvinylidine difluoride membranes (GE Healthcare). The membranes were blocked at $25^{\circ} \mathrm{C}$ with $5 \%$ skimmed milk in Tris-buffered saline (TBS) containing $0.1 \%$ Tween-20 for $1 \mathrm{~h}$ and subsequently incubated overnight at $4^{\circ} \mathrm{C}$ with the individual primary antibodies, rabbit-anti-SIRT1 (9475; 1:1,000; Cell Signaling Technology, Inc., Danvers, MA, USA), PGC-1 $\alpha$ (\#2178, 1:1,000; Cell Signaling Technology, Inc.), Bax (2772; 1:1,000; Cell Signaling Technology, Inc.), Bcl-2 (15071; 1:1,000; Cell Signaling Technology, Inc.), caspase-3 (9662; 1:1,000; IDUN Pharmaceuticals, Inc., San Diego. CA, USA), $\beta$-actin (4970; 1:1,000; Santa Cruz Biotechnology, Dallas, TX, USA). Following washing for three times with TBS-Tween for 5 min every time, the membrane was incubated with secondary antibodies of horseradish peroxidase-conjugated immunoglobulin G (Sigma-Aldrich; Merck KGaA; 1:5,000) for $1 \mathrm{~h}$ at room temperature. Following washing three times for $5 \mathrm{~min}$, the proteins were visualized with enhanced chemiluminescent substrates (GE Healthcare), and the image of each band was further quantified using Multigauge computer software version 3.0 (Berthold Australia Pty Ltd, Bundoora, Australia).

Statistical analysis. All statistical analyses were performed with SPSS software version 13.0 for windows (SPSS, Inc., Chicago, IL, USA). Data are presented as the mean \pm standard 
deviation. One-way analysis of variance with Bonferroni's correction was used to compare the statistical difference between multiple groups. The Least Significant Difference test was used for comparison between groups. $\mathrm{P}<0.05$ was considered to indicate a statistically significant difference.

\section{Results}

Effect of PSP on cell viability. Following simulation of IR conditions, the N2a cell viability was analyzed in an MTT assay (Fig. 1). Compared with the NC, the cell viability significantly decreased following IR with a survival rate of $\sim 60 \%$ (Fig. 1; $\mathrm{P}<0.05)$. Following treatment with PSP, the cell activity was significantly improved compared with the IR group and exhibited a dose-dependent manner $(\mathrm{P}<0.05)$. The cell viabilities of the PSP1, PSP2 and PSP3 groups were 84, 90 and 95\%, respectively. The data indicated that treatment with higher doses of PSP resulted in significantly higher cell viability $(\mathrm{P}<0.05)$.

Effect of PSP on cell membrane permeability. Neuronal injury or death was assessed according to levels of LDH released into the extracellular medium as previously described (24). As demonstrated in Fig. 2, the IR group exhibited a significantly higher LDH activity (170\%) compared with the NC $(\mathrm{P}<0.01)$. Following administration of PSP, the activity of LDH decreased with the concentration of PSP increasing between 10 and $50 \mathrm{mg} / \mathrm{ml}$. A high dose of PSP was able to significantly decrease the activity of LDH with the expression percentage of $105 \%$ in culture medium compared with the NC ( $\mathrm{P}<0.01$; Fig. 2), which suggests that PSP is able to reduce the cell membrane permeability and consequently reduce injury to protect cells.

Effect of PSP on caspase-3 activity in cells. The activity of caspase- 3 was determined by caspase- 3 specific substrates. The results demonstrated that the activity of caspase- 3 was significantly increased by up to $\sim 90 \%$ following IR, and decreased markedly following treatment with PSP $(\mathrm{P}<0.01$; Fig. 3). The activity of caspase-3 following treatment with PSP1, PSP2 and PSP3 was $\sim 125,120$ and 110\%, respectively. The results suggested that PSP may reduce the activity of caspase- 3 to reduce apoptosis.

Effect of PSP on neurological function score. Neurological function assessment is a crucial procedure for the medical care of neurological patients, which may detect the presence of a neurological disease or injury and monitor its progression. In the present study, each group of rats was scored as 0-3 with no subarachnoid hemorrhage observed, suggestive of successful establishment of the model. As demonstrated in Table I, the mice in the sham group had no neurological deficit symptoms. In the IR group, the rats exhibited a neurological deficit with a higher score of $2.63(\mathrm{P}<0.01)$ compared with the sham group. Compared with the IR group, the neurological deficits following treatment with PSP were reduced $(\mathrm{P}<0.05)$, particularly in the PSP 3 group $(\mathrm{P}<0.01)$. The neurological function scores of the low and middle-dose PSP groups were $2.17 \pm 0.25$ and $1.53 \pm 0.33$, respectively, which is higher compared with high-dose PSP $(1.16 \pm 0.19)$. The neurological deficit score was the lowest in the PSP3 group.

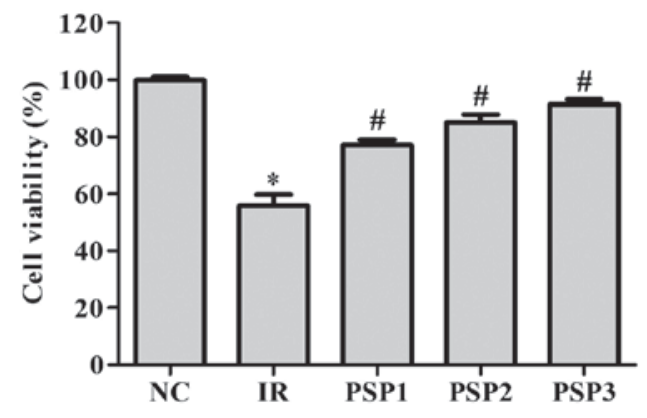

Figure 1. Effect of PSP on cell viability of N2a cells during IR injury assessed by an MTT assay $(n=6)$. The N2a cells were treated with different concentrations of PSP; $10 \mathrm{mg} / \mathrm{ml}$ (PSP1), $30 \mathrm{mg} / \mathrm{ml}$ (PSP2) and $50 \mathrm{mg} / \mathrm{ml}$ (PSP3). The data are expressed as the mean \pm standard deviation. ${ }^{~} \mathrm{P}<0.05$ vs. $\mathrm{NC} ;{ }^{\#} \mathrm{P}<0.05$ vs. IR. NC, negative control; IR, ischemia-reperfusion; PSP, polysaccharide peptide.

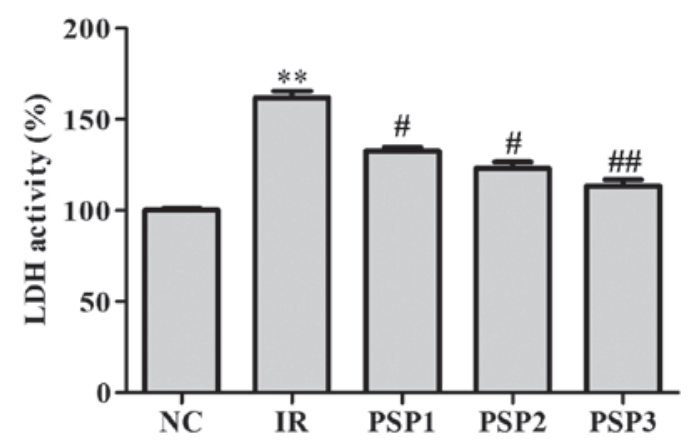

Figure 2. Effect of PSP on the release of LDH following IR injury in N2a cells $(n=3)$. The N2a cells were administrated with different concentrations of PSP; $10 \mathrm{mg} / \mathrm{ml}$ (PSP1), $30 \mathrm{mg} / \mathrm{ml}$ (PSP2) and $50 \mathrm{mg} / \mathrm{ml}$ (PSP3). The data are expressed as the mean \pm standard deviation. ${ }^{* *} \mathrm{P}<0.01$ vs. $\mathrm{NC} ;{ }^{~} \mathrm{P}<0.05$, ${ }^{\# \#} \mathrm{P}<0.01$ vs. IR. LDH, lactate dehydrogenase; NC, negative control; IR, ischemia-reperfusion; PSP, polysaccharide peptide.

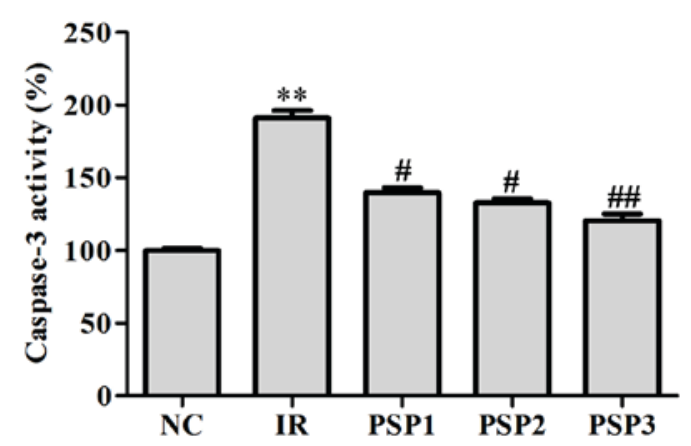

Figure 3. Effect of PSP on caspase-3 activity in N2a cells $(n=3)$. The N2a cells were administrated with different concentrations of PSP; $10 \mathrm{mg} / \mathrm{ml}$ (PSP1), $30 \mathrm{mg} / \mathrm{ml}$ (PSP2) and $50 \mathrm{mg} / \mathrm{ml}$ (PSP3). The data are expressed as the mean \pm standard deviation. ${ }^{* *} \mathrm{P}<0.01$ vs. $\mathrm{NC} ;{ }^{\#} \mathrm{P}<0.05,{ }^{\# \#} \mathrm{P}<0.01$ vs. IR. $\mathrm{NC}$, negative control; IR, ischemia reperfusion; PSP, polysaccharide peptide.

Effect of PSP on cerebral infarction volume. To assess the protective effects of PSP against brain ischemia injury in vivo, a model of ischemia was established. Infarction size was assessed by the appearance of a white region following TTC staining (Fig. 4). The results demonstrated that there was no white region observed in brain tissue in the sham group, and the infarction was observed in the IR group and 
Table I. Neural function defect scale in different treatment groups.

\begin{tabular}{lcc}
\hline Groups & $\mathrm{n}$ & Score \\
\hline Sham & 15 & 0 \\
IR & 15 & $2.63 \pm 0.48^{\mathrm{a}}$ \\
PSP1 & 15 & $2.17 \pm 0.25^{\mathrm{b}}$ \\
PSP2 & 15 & $1.53 \pm 0.33^{\mathrm{b}}$ \\
PSP3 & 15 & $1.16 \pm 0.19^{\mathrm{c}}$ \\
\hline
\end{tabular}

${ }^{\mathrm{a}} \mathrm{P}<0.01$ vs. sham group; ${ }^{\mathrm{b}} \mathrm{P}<0.05,{ }^{\mathrm{c}} \mathrm{P}<0.01$ vs. IR group. IR, ischemia reperfusion; PSP, polysaccharide peptide.

A

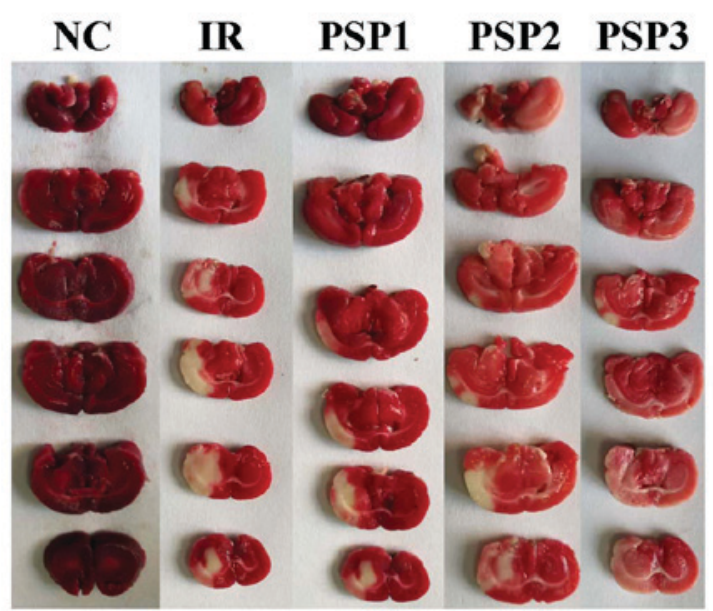

B

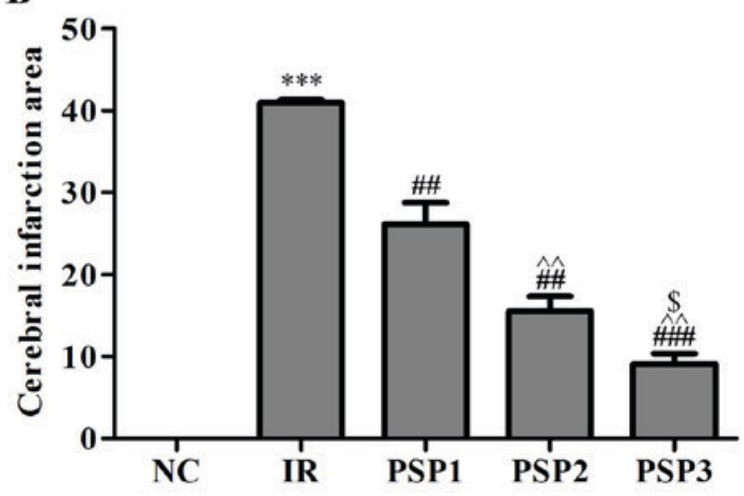

Figure 4. Alteration in cerebral infarct volume in different treatment groups. (A) PSP1 is the low dose group $(150 \mathrm{mg} / \mathrm{kg})$, PSP2 is the middle dose group (200 mg/kg) and PSP3 is the high dose group (250 mg/kg). (B) The data are expressed as the mean \pm standard deviation. ${ }^{* * * *} \mathrm{P}<0.001$ vs. $\mathrm{NC} ;{ }^{\#} \mathrm{P}<0.01$,

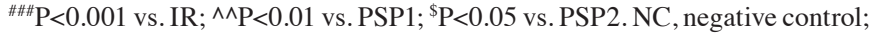
$\mathrm{IR}$, ischemia reperfusion; PSP, polysaccharide peptide.

PSP groups. Compared with the IR group (42.93\%), the infarction volume in the PSP treatment groups decreased significantly $(\mathrm{P}<0.01)$. Among the PSP administration groups, the score of PSP3 group (25.16\%) was lower compared with PSP1 (36.17\%; P<0.01) and PSP2 (33.53\%; P<0.05). In addition, there was a significant difference between low- and middle-dose group $(\mathrm{P}<0.01)$. These results indicated that PSP may effectively reduce the infarction size in a dose-dependent manner.

Histopathological evaluation. H\&E staining was used to examine the morphological alterations of the hippocampus area upon treatment with PSP following IR injury. As demonstrated in Fig. 5, the nerve cells in the sham group were structurally complete, arranged neatly, stained evenly and clearly. In the IR group, the number of nerve cells decreased, were arranged disorderly and the stain color deepened. Compared with the IR group, the number of cells in the PSP-treated groups increased, and the morphology of the nerve cells recovered well. These results indicated that PSP may reduce brain cell death in the hippocampus area caused by IR and reduce brain injury.

Effect of PSP on SIRT1, PGC-1 1 , Bax, Bcl-2 and caspase-3 protein expression. To verify whether the SIRT1 signaling pathway participates in the protection from IR injury mediated by PSP, the expression of SIRT1 and PGC-1 $\alpha$ was examined by western blotting following IR injury. As demonstrated in Fig. 6, the expression of SIRT1 and PGC-1 $\alpha$ was decreased in the IR group, with $\sim 0.3$ fold and $\sim 0.25$ fold change, respectively, compared with the sham group $(\mathrm{P}<0.01)$. Following intraperitoneal injection of different concentrations of PSP (150, 200 and $250 \mathrm{mg} / \mathrm{kg})$, the expression of SIRT1 and PGC-1 $\alpha$ was upregulated $(\mathrm{P}<0.05)$, particularly in the PSP3 group $(\sim 0.9$ fold change and $\sim 1.1$ fold change, respectively) compared with the IR group. These results demonstrated that PSP may upregulate the expression of neuroprotective factors SIRT1 and PGC-1 $\alpha$, which may protect cerebral IR injury in rats.

The effect of PSP on Bax, Bcl-2 and caspase-3 was additionally examined by western blotting (Fig. 6). Compared with the sham group, the $\mathrm{Bax} / \mathrm{Bcl}-2$ protein expression level in the IR group was significantly increased at $\sim 160 \%(\mathrm{P}<0.01)$. Compared with the IR group, the $\mathrm{Bax} / \mathrm{Bcl}-2$ protein expression in the PSP group was downregulated to $\sim 120,90$ and $68 \%$, corresponding to PSP1, PSP2 and PSP3 $(\mathrm{P}<0.05)$. It is demonstrated in Fig. 6 that the caspase-3 protein expression level in the IR group was significantly increased $(\mathrm{P}<0.01)$ compared with the sham group. However, following treatment with PSP, the expression of caspase-3 protein was significantly decreased $(\mathrm{P}<0.05)$. A dose of $250 \mathrm{mg} / \mathrm{kg}$ PSP was able to downregulate the expression of caspase-3 more effectively. These data suggested that the protection of PSP against cerebral IR in rats may be due to an increase in $\mathrm{Bcl}-2$ protein expression and a decrease in Bax and caspase- 3 protein expression, thus reducing the apoptosis rate and reducing injury.

\section{Discussion}

In the present study, the cell viability, and the activity of LDH and caspase- 3 were examined by an MTT assay and ELISA in vitro. A similar environment of cerebral IR injury was stimulated for the N2a cells. It was identified that higher concentrations of PSP markedly improved the cell viability of the N2a cells. PSP reduced the activity of LDH and caspase-3. Therefore, the in vitro study suggested that PSP may be able to improve cell viability, increase the expression of caspase-3 protein and protect N2a cells against cerebral IR injury in vitro. 

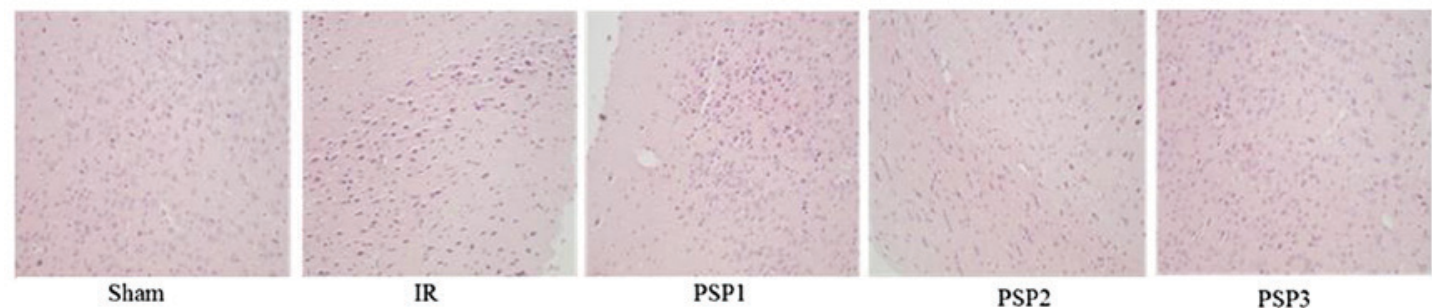

Figure 5. Hematoxylin and eosin staining of ischemic hippocampus in the sham, IR, PSP1, PSP2 and PSP3 groups. PSP1 is the low dose group (150 mg/kg), PSP2 is the middle dose group (200 mg/kg) and PSP3 is the high dose group $(250 \mathrm{mg} / \mathrm{kg})$. Magnification, $\mathrm{x} 100$. PSP, polysaccharide peptide; IR, ischemia reperfusion.
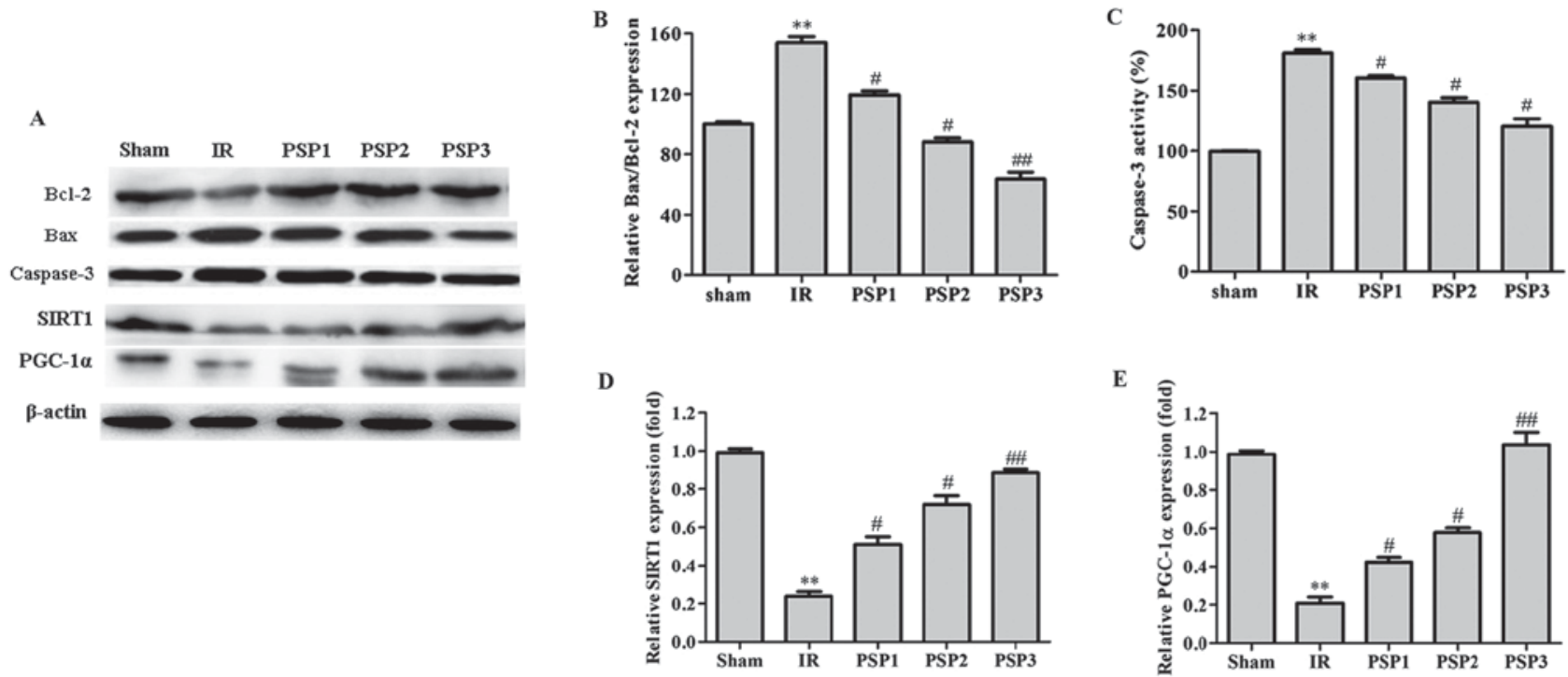

Figure 6. Effect of PSP on the expression of related proteins (Bcl-2, Bax and caspase-3, SIRT1 and PGC-1 $\alpha$ ) in the ischemic hippocampus of rats in each group. (A) The protein analyzed by western blotting. (B) The ratio of Bax to Bcl protein level. The quantitative graph of (C) caspase-3, (D) SIRT1 and (E) PGC-1 $\alpha$ protein levels. PSP1 is the low dose group (150 mg/kg), PSP2 is the middle dose group ( $200 \mathrm{mg} / \mathrm{kg}$ ) and PSP3 is the high dose group ( $250 \mathrm{mg} / \mathrm{kg})$. The data are expressed as the mean \pm standard deviation. ${ }^{* *} \mathrm{P}<0.01$ vs. respective sham; ${ }^{~} \mathrm{P}<0.05,{ }^{\# \#} \mathrm{P}<0.01$ vs. respective IR. PGC-1 $\alpha$, peroxisome proliferator-activated receptor $\gamma$ coactivator-1 $\alpha$; Bcl-2, B-cell lymphoma 2; Bax, Bcl-2-like protein 4; SIRT1, silent information regulator protein 1; PGC-1 $\alpha$, peroxisome proliferator-activated receptor $\gamma$ coactivator-1 $\alpha$; PSP, polysaccharide peptide; IR, ischemia reperfusion.

Following establishment of an IR model in SD rats, the PSP effect on neuroprotection was examined in vivo. It is reported that focal cerebral ischemia may cause cerebral cell death, and subsequently result in neuronal deficit symptoms and local infarction (25). The neuronal deficit severity and infarction area indicate the grade of brain injury (26). In the animal experiments, treatment with PSP exhibited neuroprotective effects, demonstrated by alleviation of histological injury in the ischemic cortex and neurological deficits derived from IR injury in rats. All treatment groups with different doses of PSP has reduced the cerebral infraction and improved neurological scores at $24 \mathrm{~h}$ post reperfusion, and the most marked effect of PSP was identified in the group treated with $250 \mathrm{mg} / \mathrm{kg}$ PSP. Therefore, a higher concentration of PSP was used to evaluate the protection and its side effect on the other functions. The morphology of infarct nerve cells determined by H\&E staining the neuroprotection mediated by PSP. The results of the $\mathrm{H} \& \mathrm{E}$ staining and the caspase- 3 activity assay revealed the neuroprotective effect of PSP was associated with a reduction of neuronal loss and apoptosis induced by IR injury.

During the pathological process of cerebral IR injury, apoptosis in neurons serves a key role, according to a previous study (4). Apoptosis is controlled cell death regulated by the cell's natural self-destruction. In the process of apoptosis, specific caspase enzymes affect proteins that have crucial functions (27). At present, two pathways of apoptosis have been well documented: The extrinsic and intrinsic pathways. The extrinsic pathway is initiated by extracellular insult and predominately relies on caspase- 8 activation. A cascade of proteases are activated by caspase- 8 , including caspase- 9 and caspase-7, to trigger caspase-3, which executes apoptosis (28). Mechanically, caspase-3 is able to cleave itself and affect the other key proteins, including the DNA breaks of canonical apoptosis (29). Therefore, activation and expression of caspase- 3 promotes cell apoptosis. In the normal mouse brain, caspase- 3 expression is at lower level (30). In the present study, caspase-3 activity was increased significantly following IR, and decreased markedly following treatment with PSP for $24 \mathrm{~h}$.

The intrinsic pathway, which is also termed the mitochondrial pathway due to the essential role of mitochondria, is initiated by Bax. Bax translocates to the mitochondrial membrane from the cytosol and competes with other members of the Bcl-2 family. Therefore, the activation of the intrinsic 
pathway is associated with a ratio of Bcl-2 and Bax. An imbalance between Bcl-2 and Bax results in the activation of caspase-9 (31). To examine the mechanisms that are altered by PSP in the IR injury model, the Bcl-2/Bax ratio was assessed. The present results suggested a significant difference in $\mathrm{Bax} / \mathrm{Bcl}-2$ ratio between the sham group and IR group. The $\mathrm{Bax} / \mathrm{Bcl}-2$ ratio in the IR group reached its highest value after $22 \mathrm{~h}$ of reperfusion. However, the $\mathrm{Bax} / \mathrm{Bcl}-2$ ratio was reverted and decreased following treatment with PSP, as the decreased Bax/Bcl-2 ratio may prevent neuronal cell apoptosis by impeding the Bax/Bax homodimer formation and promoting Bcl-2/Bax heterodimer formation. Thus, the present results again indicated that treatment with PSP following IR injury may protect neuronal cells from apoptosis.

SIRT1, a homologue of silent information regulator (Sir2) protein, is the closest mammalian homologue of yeast Sir2. It is classified as a NAD-dependent deacetylase and a nuclear sirtuin, although it is not restricted to the nucleus and has important non-nuclear functions (32). SIRT1 is a key factor in apoptotic cell death, cellular senescence and vascular growth associated with IR (33). SIRT1 physically interacts with and deacetylates PGC-1 $\alpha$ at multiple lysine sites, resulting in increased activity of PGC-1 $\alpha$. Xia et al (34) reported that SIRT1 and AMP-activated protein kinase (AMPK) were involved in the stimulation of nitric oxide (NO) production by endothelial NO synthase and enhancement of NO bioavailability, which may be an important mechanism associated with the protective effect of SIRT1 in IR injury. Feng et al (35) demonstrated that treatment with bakuchiol attenuates IR injury by reducing IR-induced mitochondrial oxidative damage via the activation of SIRT1/PGC-1 $\alpha$ signaling. The SIRT1/AMPK pathway is involved in the protection induced by a number of agents, including aspirin (36), resveratrol (37). Notably, SIRT1 and AMPK expression were reported to be increased by heat shock protein 70 (HSP70) overexpression (38). The present study additionally demonstrated that the levels of SIRT1 and PGC- $1 \alpha$ protein were elevated in all three PSP groups. The next step will be to examine whether HSP70 also has a role in cerebral IR injury, and the association of HSP70, SIRT1 and PGC-1 $\alpha$ and Bcl-2/Bax, and, subsequently, the effect on cerebral IR injury by the intervention of these factors. These results indicated that the mechanism of seaweed-derived PSP protection of the brain from IR injury may be attributed to these alterations and their associated signaling pathway.

In the present study, the results revealed that the protective effect of seaweed polysaccharide on cerebral IR injury in vitro and in vivo and the mechanism may be associated with its effect on the regulation of the SIRT1/PGC- $1 \alpha$ signaling pathway. Thus, application of seaweed polysaccharide may provide a novel promising approach for cerebral IR injury therapy.

\section{Acknowledgements}

Not applicable.

\section{Funding}

No funding was received.

\section{Availability of data and materials}

All data generated or analyzed during this study are included in this published article.

\section{Authors' contributions}

PX and DW participated in the conception and design of the study. PX and KM conducted the experiments and data acquisition. JW and WL conducted the histological examination of the brain tissue and analyzed and interpreted the data. PX was a major contributor in writing the manuscript. DW revised the manuscript and provide the final approval of the submitted version.

\section{Ethics approval and consent to participate}

All the animal works were performed stringently according to the regulation of the Guide for the Care and Use of Laboratory Animals in China. The protocol was approved by the Committee of the Ethics of Animal Experiments of Shanghai Jiaotong University Affiliated Sixth Hospital Provincial Centre for Disease Control and Prevention (proposal authorization no. SYXK/2016/050122).

\section{Patient consent for publication}

Not applicable.

\section{Competing interests}

The authors declare that they have no competing interests.

\section{References}

1. Yamashita $\mathrm{T}$ and Abe K: Recent progress in therapeutic strategies for ischemic stroke. Cell Transplant 25: 893-898, 2016.

2. Dong Q, Lin X, Shen L and Feng Y: The protective effect of herbal polysaccharides on ischemia-reperfusion injury. Int J Biol Macromol 92: 431-440, 2016.

3. Rao PR, Kumar VK, Viswanath RK and Subbaraju GV: Cardioprotective activity of alcoholic extract of Tinospora cordifolia in ischemia reperfusion induced myocardial infarction in rats. Biol Pharm Bull 28: 2319-2322, 2005.

4. Kalogeris T, Baines CP, Krenz M and Korthuis RJ: Cell biology of ischemia/reperfusion injury. Int Rev Cell Mol Biol 298: 229-317, 2012.

5. Zerna C, Assis Z, d'Esterre CD, Menon BK and Goyal M: Imaging, intervention, and workflow in acute ischemic stroke: The calgaryapproach. AJNR Am J Neuroradiol 37: 978-984, 2016.

6. Sugawara T and Chan PH: Reactive oxygen radicals and pathogenesis of neuronal death after cerebral ischemia. Antioxid Redox Signal 5: 597-607, 2003.

7. Muralikrishna Adibhatla R and Hatcher JF: Phospholipase A2, reactive oxygen species, and lipid peroxidation in cerebral ischemia. Free Radic Biol Med 40: 376-387, 2006.

8. Xu M, Wang HF, Zhang YY and Zhuang HW: Protection of rats spinal cord ischemia-reperfusion injury by inhibition of MiR497 on inflammation and apoptosis: Possible role in pediatrics. Biomed Pharmacother 81: 337-344, 2016.

9. Zhu T, Yao Q, Hu X, Chen C, Yao H and Chao J: The role of MCPIP1 in ischemia/reperfusion injury-induced HUVEC migrationand apoptosis. Cell Physiol Biochem 37: 577-591, 2015.

10. Arda-Pirincci P and Bolkent S: The role of epidermal growth factor in prevention of oxidative injury and apoptosis induced by intestinal ischemia/reperfusion in rats. Acta Histochem 116: 167-175, 2014. 
11. Shokeir AA, Barakat N, Hussein AM, Awadalla A, Harraz AM, Khater S, Hemmaid K and Kamal AI: Activation of Nrf2 by ischemic preconditioning and sulforaphane in renalischemia/reperfusion injury: A comparative experimental study. Physiol Res 64: 313-323, 2015.

12. Ju J, Wu J and Hou R: Role of the p38 mitogen-activated protein kinase signaling pathway inestrogen-mediated protection following flap ischemia-reperfusion injury. Cell Biochem Funct 34: 522-530, 2016.

13. Kovalska M, Kovalska L, Pavlikova M, Janickova M, Mikuskova K, Adamkov M, Kaplan P, Tatarkova Z and Lehotsky J: Intracellular signaling MAPK pathway after cerebral ischemia-reperfusion injury. Neurochem Res 37: 1568-1577, 2012.

14. Imahashi K, Schneider MD, Steenbergen C and Murphy E: Transgenic expression of Bcl-2 modulates energy metabolism, preventscytosolic acidification during ischemia, and reduces ischemia/reperfusion injury. Circ Res 95: 734-741, 2004

15. Wei DF, Chen T, Yan M, Zhao W, Li F, Cheng W and Yuan L: Synthesis, characterization, antioxditant activity and neuroprotective effects of selenium polysaccharide from Radix hedysari. Carbohydr Polym 125: 161-168, 2015.

16. Sanagi MM, Loh SH, Wan Ibrahim WN, Pourmand N, Salisu A, Wan Ibrahim WA and Ali I: Agarose- and alginate based biopolymers for sample preparation: Excellentgreen extraction tools for this century. J Sep Sci 39: 1152-1159, 2016.

17. Gong J, Sun F, Li Y, Zhou X, Duan Z, Duan F, Zhao L, Chen H, Qi S and Shen J: Momordica charantia polysaccharides could protect against cerebral ischemia/reperfusion injury through inhibiting oxidative stress mediated c-Jun $\mathrm{N}$-terminal kinase 3 signaling pathway. Neuropharmacology 91: 123-134, 2015.

18. Zhou ZY, Tang YP, Xiang J, Wua P, Jin HM, Wang Z, Mori M and Cai DF: Neuroprotective effects of water-soluble ganoderma lucidum polysaccharideson cerebral ischemic injury in rats J Ethno pharmacol 131: 154-164, 2010.

19. Lu ZQ, Deng YJ and Lu JX: Effect of aloe polysaccharide on caspase-3 expression following cerebral ischemia and reperfusion injury in rats. Mol Med Rep 6: 371-374, 2012.

20. Zhang S, He B, Ge J, Zhai C, Liu X and Liu P: Characterization of chemical composition of agaricus brasiliensis polysaccharides and its effect on myocardial SOD activity, MDA and caspase-3 level in ischemia-reperfusion rats. Int J Biol Macromol 46: 363-366, 2010

21. Reis C, Wang Y, Akyol O, Ho WM, Ii RA, Stier G, Martin R and Zhang JH: What's new in traumatic brain injury: Update on tracking, monitoring and treatment. Int J Mol Sci 16 : 11903-11965, 2015.

22. Shi P, Zhang L, Wang J, Lu D, Li Y, Ren J, Shen M, Zhang L and Huang J: Porcine FceRI mediates porcine reproductive and respiratory syndrome virus multiplication and regulates the inflammatory reaction. Virol Sin 33: 249-260, 2018.

23. Bösel J, Ruscher K, Ploner CJ and Valdueza JM: Delayed neurological deterioration in a stroke patient with postoperative acute anemia. Eur Neurol 53: 36-38, 2005.
24. Haigis MC and Sinclair DA: Mammalian sirtuins: Biological insights and disease relevance. Annu Rev Pathol 5: 253-295, 2010.

25. Zille M, Farr TD, Przesdzing I, Müller J, Sommer C, Dirnagl U and Wunder A: Visualizing cell death in experimental focal cerebral ischemia: Promises, problems, and perspectives. J Cereb Blood Flow Metab 32: 213-231, 2012.

26. Hyman BT and Yuan J: Apoptotic and non apoptotic roles of caspases in neuronal physiology and pathophysiology. Nat Rev Neurosci 13: 395-406, 2012.

27. Elmore S: Apoptosis: A review of programmed cell death. Toxicol Pathol 35: 495-516, 2007.

28. Walsh JG, Cullen SP, Sheridan C, Lüthi AU, Gerner C and Martin SJ: Executioner caspase-3 and caspase-7 are functionally distinct proteases. Proc Natl Acad Sci USA 105: 12815-12819, 2008.

29. Manabat C, Han BH, Wendland M, Derugin N, Fox CK, Choi J, Holtzman DM, Ferriero DM and Vexler ZS: Reperfusion differentially induces caspase-3 activation in ischemic core and penumbra after stroke in immature brain. Stroke 34: 207-213, 2003.

30. Ferrer I and Planas AM: Signaling of cell death and cell survival following focal cerebral ischemi: Lifeand death struggle in the penumbra. J Neuropathol Exp Neurol 62: 329-339, 2003.

31. Potente M and Dimmeler S: Emerging roles of SIRT1 in vascular endothelial homeostasis. Cell Cycle 7: 2117-2122, 2008.

32. Maiese K, Chong ZZ, Shang YC and Wang S: Translating cell survival and cell longevity into treatment strategies with SIRT1. Rom J Morphol Embryol 52: 1173-1185, 2011.

33. Rodgers JT, Lerin C, Haas W, Gygi SP, Spiegelman BM and Puigserver P: Nutrient control of glucose homeostasis through a complex of PGC-1alpha and SIRT1. Nature 434: 113-118, 2005.

34. Xia N, Förstermann U and Li HG: Resveratrol and endothelial nitric oxide. Molecules 19: 16102-16121, 2014.

35. Feng J, Yang Y, Zhou Y, Wang B, Xiong H, Fan C, Jiang S, Liu J, Ma Z, Hu W, et al: Bakuchiol attenuates myocardial ischemia reperfusion injury by maintaining mitochondrial function: The role of silent information regulator 1. Apoptosis 21: 532-545, 2016.

36. Tsai KL, Huang PH, Kao CL, Leu HB, Cheng YH, Liao YW, Yang YP, Chien Y, Wang CY, Hsiao CY, et al: Aspirin attenuates vinorelbine-induced endothelial inflammation via modulating SIRT1/AMPK axis. Biochem Pharmacol 88: 189-200, 2014.

37. Tamaki N, Cristina Orihuela-Campos R, Inagaki Y, Fukui M, Nagata T and Ito HO: Resveratrol improves oxidative stress and prevents the progression of periodontitis via the activation of the Sirt1/AMPK and the Nrf2/antioxidant defense pathways in a rat periodontitis model. Free Radic Biol Med 75: 222-229, 2014.

38. Liu S, Xu J, Fang C, Shi C, Zhang X, Yu B and Yin Y: Over-expression of heat shock protein 70 protects mice against lung ischemia/reperfusion injury through SIRT1/AMPK/eNOS pathway. Am J Transl Res 8: 4394-4404, 2016.

This work is licensed under a Creative Commons Attribution-NonCommercial-NoDerivatives 4.0 International (CC BY-NC-ND 4.0) License. 